\title{
Factors Associated with Medication Nonadherence among Hypertensives in Ghana and Nigeria
}

\author{
Vincent Boima, ${ }^{1,2}$ Adebowale Dele Ademola, ${ }^{3,4}$ Aina Olufemi Odusola, \\ Francis Agyekum, ${ }^{2}$ Chibuike Eze Nwafor, ${ }^{6}$ Helen Cole, ${ }^{7}$ Babatunde L. Salako, ${ }^{8,9}$ \\ Gbenga Ogedegbe, ${ }^{7,10}$ and Bamidele O. Tayo ${ }^{11}$ \\ ${ }^{1}$ School of Medicine and Dentistry, University of Ghana, P.O. Box 4236, Accra, Ghana \\ ${ }^{2}$ Department of Medicine, Korle Bu Teaching Hospital, P.O. Box 77, Accra, Ghana \\ ${ }^{3}$ Department of Paediatrics, Faculty of Clinical Sciences, College of Medicine, University of Ibadan, Ibadan PMB 5017, \\ Oyo State, Nigeria \\ ${ }^{4}$ Department of Paediatrics, University College Hospital, Ibadan PMB 5116, Oyo State, Nigeria \\ ${ }^{5}$ Department of Medicine, General Hospital, Randle Avenue, Apapa PMB 1014, Lagos, Nigeria \\ ${ }^{6}$ Cardiology Division, Department of Medicine, University of Port Harcourt Teaching Hospital, Port Harcourt PMB 6173, \\ Rivers State, Nigeria \\ ${ }^{7}$ Department of Population Health, NYU School of Medicine, 227 E. 30th Street, 6th Floor, New York, NY 10016, USA \\ ${ }^{8}$ Department of Medicine, Faculty of Clinical Sciences, College of Medicine, University of Ibadan, Ibadan PMB 5017, Oyo State, Nigeria \\ ${ }^{9}$ Department of Medicine, University College Hospital, Ibadan PMB 5116, Oyo State, Nigeria \\ ${ }^{10}$ Global Institute of Public Health, New York University, New York, NY 10003, USA \\ ${ }^{11}$ Department of Public Health Sciences, Stritch School of Medicine, Loyola University Chicago, 2160 South First Avenue, \\ Maywood, IL 60153, USA
}

Correspondence should be addressed to Adebowale Dele Ademola; dr_deboademola@yahoo.co.uk

Received 13 December 2014; Accepted 25 March 2015

Academic Editor: Francesco Cappuccio

Copyright (C) 2015 Vincent Boima et al. This is an open access article distributed under the Creative Commons Attribution License, which permits unrestricted use, distribution, and reproduction in any medium, provided the original work is properly cited.

Background. Blood pressure (BP) control is poor among hypertensives in many parts of sub-Saharan Africa. A potentially modifiable factor for control of BP is medication nonadherence (MNA); our study therefore aimed to determine factors associated with MNA among hypertensives in Ghana and Nigeria. Methodology. We conducted a multicenter cross-sectional study. Patients were recruited from Korle-Bu Hospital $(n=120)$, Ghana; and University of Port Harcourt Teaching Hospital, $(n=73)$ Apapa General Hospital Lagos $(n=79)$ and University College Hospital Ibadan $(n=85)$, Nigeria. Results. 357 hypertensive patients $(42.6 \%$ males) participated. MNA was found in $66.7 \%$. Adherence showed correlation with depression $(r=-0.208, P<0.001)$, concern about medications $(r=-0.0347, P=0.002)$, and knowledge of hypertension $(r=0.14, P=0.006)$. MNA was associated with formal education $(P=0.001)$ and use of herbal preparation $(P=0.014)$. MNA was found in $61.7 \%$ of uninsured participants versus $73.1 \%$ of insured participants $(P=0.032)$. Poor BP control was observed in $69.7 \%$ and there was significant association between MNA and poor BP control $(P=0.006)$. Conclusion. MNA is high among hypertensives in Ghana and Nigeria and is associated with depression, concern about hypertensive medications, formal education, and use of herbal preparations. The negative association between health insurance and MNA suggests interplay of other factors and needs further investigation.

\section{Introduction}

Hypertension is a common but treatable public health problem globally. It is estimated to cause 7.5 million deaths annually, about $12.8 \%$ of all deaths worldwide [1]. Globally, the prevalence of hypertension in adults was approximately $40 \%$ in 2008 [2]. The number of people worldwide with hypertension rose from 600 million in 1980 to nearly 1 billion in 2008. The burden of hypertension is particularly high in sub-Saharan African countries. The reported prevalence of 
hypertension in Ghana ranges from $19 \%$ to $32.8 \%$ in rural areas and 25.5 to $48 \%$ in urban areas [3-5]. Similarly, recent studies in Nigeria showed that the prevalence of hypertension in rural areas ranges from 21 to $25 \%$ [6, 7] while in semiurban and urban areas the prevalence ranged from 27 to $46 \%$ [811]. Optimal blood pressure (BP) control with levels below $140 / 90 \mathrm{mmHg}$ is associated with significant reduction in cardiovascular complications such as stroke and coronary heart disease $[12,13]$.

The increasing rates of hypertension in sub-Saharan Africa have been attributed to rapid epidemiologic transition from an agrarian lifestyle to a more westernized lifestyle, with increasing rates of obesity, unhealthy diet, and physical inactivity $[3,14]$. Blood pressure control is generally poor among hypertensive patients in sub-Saharan Africa, and efforts to improve BP control are needed. Poor BP control among hypertensives in sub-Saharan Africa is related to the complex interplay of patient, provider, and socioeconomic factors in this region $[3,14]$. For example, patients may lack knowledge about hypertension, or they may harbour beliefs that are discordant with those of the traditional medical model regarding the causes and treatment of hypertension. As a result, patients' beliefs may be discordant with practices associated with BP control potentially leading to poor medication adherence. Additionally, patients may exhibit poor medication adherence because they are unable to afford the cost of medications. Similarly, healthcare providers may have insufficient time and resources to provide the necessary education and treatment.

Of the patient factors that affect BP control, poor medication adherence is the most salient and little studied in sub-Saharan African countries including Nigeria and Ghana. Medication nonadherence (MNA) is a potentially modifiable risk factor for the improvement of BP control. Factors that may influence adherence include socioeconomic status, access to health insurance, depression, patient's knowledge of the disease, and beliefs about medications [15]. Past studies have reported a wide range of MNA in Ghana and Nigeria from 17.4 to $93 \%$, making it difficult to compare the degree and factors associated with MNA in both countries [16-24].

In order to address these significant gaps in the adherence literature in sub-Saharan Africa, our study documents the levels and factors associated with MNA among hypertensive patients in the hospital setting in Ghana and Nigeria.

\section{Methods}

In this multicenter cross-sectional study, patients were recruited from four hospitals: Korle-Bu Hospital $(n=120)$, Ghana, and University of Port Harcourt Teaching Hospital, $(n=73)$ Apapa General Hospital Lagos $(n=79)$, and University College Hospital Ibadan $(n=85)$, Nigeria. Patients were eligible to be in this study if they were aged 18 years or older, were diagnosed hypertensive, and had been placed on medication for at least twelve months and provided informed consent. Hypertension was defined as systolic BP of $\geq 140 \mathrm{mmHg}$ and/or diastolic BP of $\geq 90 \mathrm{mmHg}$ [25] or patients already under treatment with antihypertensive medications. Patients were recruited from general outpatient or specialist medical clinics. The questionnaires were administered by the authors or trained research assistants. At each site, each consecutive patient who met the inclusion criteria was recruited. The same recruitment procedure was used in each center. Patient recruitment into the study took place between April and September 2013. Data collection was conducted by the authors and trained research assistants at each study site using a structured questionnaire. Blood pressure was measured with an Omron electronic BP machine, after at least 10 minutes rest, in the dominant arm of seated patients on three occasions at an interval of one minute. The average of the last 2 readings was recorded.

The dependent variables were medication adherence and BP control. Adherence to antihypertensive medications was measured using the 8-item Morisky medication adherence scale. Based on a pilot study in Korle Bu Ghana, the response options for the Morisky scale were modified from "never/rarely," "once in a while," "sometimes," "usually," and "all the time" to "Never/rarely" versus "sometimes/usually/all the time." Individual item scores were summed and those with a score of 8 were considered adherent to medication. Medium adherence corresponded to a score of 6-8, while a score of $<6$ was considered low adherence [26]. Medication nonadherence referred to a score of $<8$ (i.e., medium or low adherence). Blood pressure control was defined as systolic $\mathrm{BP}<140 \mathrm{mmHg}$ and diastolic $\mathrm{BP}<90 \mathrm{mmHg}$ [27].

Demographic and socioeconomic characteristics including age, gender, ethnicity, education, occupation, and income were recorded. Educational status was classified as none, primary, junior secondary, senior secondary, and tertiary. Monthly income was stratified as $<\$ 100, \$ 101-300, \$ 301-1000$, $\$ 1001-3000$, and $>\$ 3000$. Clinical information regarding when the diagnosis of hypertension was made, for how long patients had been on antihypertensives, and presence of any associated comorbid conditions were obtained from patients' medical records. Beliefs about medication were measured using the modified beliefs about medication questionnaire (BMQ) [28]. The BMQ includes questions on beliefs about the necessity of taking medications (BMQ-necessity) and concerns about medications (BMQ-concern). The difference between the BMQ-necessity and BMQ-concern scores was also determined for each patient (BMQ necessity-concern score). Each question on the BMQ is based on a 5-point Likert scale with response options: "strongly agree," "agree," "disagree," "strongly disagree," and "uncertain." Pilot testing of the BMQ indicated that participants were more comfortable with 3 response options. We therefore modified the responses to "agree," "disagree," and "uncertain," The parameters were scored as disagree $=1$, uncertain $=2$, and agree $=3$. The sum total of the BMQ-necessity score, BMQ-concern, and the difference between BMQ-necessity and concern scores was calculated [28]. The Patient Health Questionnaire 9 (PHQ9) was used to assess depression in this study. For this scale, sores of $0,1,2$, and 3 are assigned to response categories of "not at all," "several days," "more than half the days," and "nearly every day," respectively. The PHQ-9 total score ranges from 0 to 27. PHQ-9 scores of 0-4, 5-9, 10-14, 15-19, and 2027 represent none to minimal, mild, moderate, moderately severe, and severe depression, respectively. Scores of $>4$ were 
classified as depression. A 17-item questionnaire based on the JNC 7 and adapted from previous studies was used to assess knowledge of hypertension [14, 29-31]. The questions were based on true or false responses to statements such as "high blood pressure cannot be cured but can be controlled," "high blood pressure can damage hearts," "a person who has high blood pressure should take more salt," and "management of high blood pressure must include medications, diet, and lifestyle modification." A score of 1 mark was given for each correct response and the final score was a sum total of the correct responses.

Data collected was entered into Statistical Package for Social Sciences (SPSS) software student version 17.0 and used for analysis. Descriptive statistics such as means, standard deviations, proportions, and percentages were used to summarize quantitative and qualitative variables, respectively. Inferential statistics including Chi-square analysis and Pearson's correlation were used to compute associations between variables. The student $t$-test was used to compare means. $P$ values $<0.05$ were considered significant and $r$-values of $>0.15$ were considered strong correlations. Ethical approval for the study was obtained from University of Ghana Ethical and Protocol Review Committee (EPRC), the University of Ibadan/University College Hospital Ibadan Ethical Review Committee, and the University of Port Harcourt Ethical Review Committee.

\section{Results}

As shown in Table 1, a total of 357 hypertensive patients were recruited for the study, of which $42.6 \%$ were men. Their mean age was $56.6 \pm 13.2$ years. The majority of the study participants $(33.61 \%)$ were recruited from Korle $\mathrm{Bu}$, while $20.4 \%, 22.1 \%$, and $23.8 \%$ participants were recruited from Port Harcourt, Apapa, and Ibadan, respectively. There were significant differences in the age of participants, gender, and access to health insurance between sites. The cohort from Korle $\mathrm{Bu}$ had the highest proportion of females and participants who were more likely to have health insurance.

Nonadherence to medications was present in $66.7 \%$ of participants. Port Harcourt had the highest prevalence of MNA (95.9\%), while Ibadan had the lowest (45.1\%). Depression was present in $31.4 \%$ of the study participants, with $2.8 \%$ and $1.4 \%$ reporting moderately severe and severe depression, respectively. Depression was more prevalent in the cohort from Korle Bu (41.7\%) and Port Harcourt (41.1\%) compared to the other centres. Table 1 shows the distribution of patient characteristics by site.

3.1. Relationship among Depression, Beliefs about Medicines, and Medication Adherence. The mean age of patients who were nonadherent to medications was $54.5 \pm 13.2$ years while those who were adherent had a mean age of $60.9 \pm 12.1$ years $(P<0.001)$. Among the cohort from Korle $\mathrm{Bu}$, those who were nonadherent were also significantly younger than subjects who were adherent, $55.3 \pm 13.4$ years versus $61.3 \pm 13.7$ years $(P=0.037)$. There was significant correlation between PHQ-9 score for depression and Morisky score in Korle Bu $(r=-0.230, P=0.012)$ and in the pooled data $(r=-0.203$,
$P<0.001)$. The mean BMQ-concern score among participants who were nonadherent was $9.0 \pm 2.7$ compared to $7.5 \pm 2.5$ among those who were adherent. There was a significant negative correlation between BMQ-concern score and Morisky score in Ibadan $(r=-0.338, P=0.002)$ and in the pooled data $(r=-0.355, P=0.000)$. The BMQ-necessityconcern score was associated with MNA in the pooled data $(r=0.336, P<0.001)$. Knowledge of hypertension was significantly but weakly correlated with Morisky score $(P=$ $0.006, r=0.14)$. Table 2 shows correlations among Morisky score and depression, beliefs about medication, knowledge of hypertension, and income by site.

3.2. Relationship among Socioeconomic Status, Income, Health Insurance, and Medication Adherence. Level of income did not show correlation with Morisky score $(r=0.021, P=$ 0.744). The majority of participants $(56.3 \%)$ did not have any health insurance. MNA occurred in $47.7 \%$ of those who did not receive formal education compared with $70.9 \%$ of those who had some form of formal education $(P=0.001)$. MNA was noted in $61.7 \%$ of those who did not have health insurance and in $73.1 \%$ of those who had health insurance $(P=0.032)$. MNA was significantly more common among patients who used herbal preparation for the treatment of systemic hypertension $(P=0.014)$. There were no differences in medication adherence between patients who were married compared with those who were not. See Table 3 for a summary of characteristics by adherence.

3.3. Correlations of Blood Pressure Control. Blood pressure was controlled in $35.9 \%$ of study participants. There was variation in the proportion of patients with $\mathrm{BP}$ control across the study sites ranging from 9.6\%, in Port Harcourt, to $54.1 \%$ in Ibadan. There was significant association between medication nonadherence and poor BP control in the pooled data $(P=0.006)$. Table 1 includes rates of $\mathrm{BP}$ control and medication adherence across sites. The cohort of patients from Port Harcourt had the highest level of MNA (95.9\%) and the lowest proportion with BP control $(9.6 \%, n=4)$.

\section{Discussion}

We report a high prevalence of MNA (66.7\%) among hypertensives in hospitals in Ghana and Nigeria. Patients who were nonadherent were significantly younger than patients who were adherent. There was significant negative correlation between depression and concerns about medication and medication nonadherence. Knowledge of hypertension was positively correlated with adherence. Patients who were adherent were less likely to use herbal preparations or to have received formal education and were more likely to have children. Level of income was not associated with adherence. Not unexpectedly, poor BP control was significantly associated with MNA. Access to health insurance surprisingly showed significant association with MNA. The proportion of patients who had uncontrolled BP was higher among patients who had health insurance, compared to patients without health insurance, but the finding was not significant. Further studies on the operation of the health insurance system are 
TABLE 1: Distribution of study population characteristics by site.

\begin{tabular}{|c|c|c|c|c|c|}
\hline Parameter & Korle $\mathrm{Bu}$ & P. Harcourt & Lagos & Ibadan & Pooled \\
\hline Total (\%) & $120(33.6)$ & $73(20.4)$ & $79(22.1)$ & $85(23.8)$ & $357(100)$ \\
\hline Age (years) ${ }^{\mathrm{a}}$ & $57.0 \pm 13.7$ & $47.4 \pm 12.5$ & $57.8 \pm 9.8$ & $62.9 \pm 11.5$ & $56.6 \pm 13.2$ \\
\hline Females $(\%)^{\mathrm{a}}$ & $70(58.3)$ & $37(50.7)$ & $56(70.9)$ & $42(49.4)$ & $205(57.5)$ \\
\hline Insurance $N(\%)^{\mathrm{a}}$ & $114(95)$ & $20(27.4)$ & $5(6.3)$ & $17(20)$ & $156(43.7)$ \\
\hline HTN with comorbidities (\%) ${ }^{\mathrm{a}}$ & 57.4 & 20.5 & 34.2 & 62.4 & 45.8 \\
\hline \multicolumn{6}{|l|}{ Depression (\%) } \\
\hline No $(\%)$ & $70(58.3)$ & $43(58.9)$ & $60(75.9)$ & $72(84.7)$ & $245(68.6)$ \\
\hline Mild (\%) & $32(26.7)$ & $18(24.7)$ & $18(22.8)$ & $10(11.8)$ & $78(21.8)$ \\
\hline Moderate (\%) & $10(8.3)$ & $5(6.8)$ & $1(1.3)$ & $3(3.5)$ & $19(5.3)$ \\
\hline Mord-severe (\%) & $4(3.3)$ & $6(8.2)$ & - & - & $10(2.8)$ \\
\hline Severe (\%) & $4(3.3)$ & $1(1.4)$ & - & - & $5(1.4)$ \\
\hline Total (\%) & $120(100)$ & $73(100)$ & $79(100)$ & $85(100)$ & $357(100)$ \\
\hline BMQ-necessity [median (IQR)] & $14(11-15)$ & $11(9-13)$ & $15(13-15)$ & $10.5(7-13)$ & \\
\hline BMQ-concern [median (IQR)] & $7.5(5-11)$ & $11(9-12.5)$ & $7(8-9)$ & $7(5-9)$ & \\
\hline Knowledge about HTN [median (IQR)] & $15(14-16)$ & $13(11-14)$ & $13(12-15)$ & $14(13-15)$ & $14(13-15)$ \\
\hline Nonadherence (\%) & 72.5 & 95.9 & 53.2 & 45.9 & 66.7 \\
\hline Medium adherence (\%) & 41.7 & 12.3 & 38.0 & 32.9 & 32.8 \\
\hline Low adherence (\%) & 30.8 & 83.6 & 15.2 & 12.9 & 33.9 \\
\hline Blood pressure control $(\%)^{\mathrm{a}}$ & 27.7 & 9.6 & 53.2 & 54.1 & 35.9 \\
\hline \multicolumn{6}{|l|}{ Educational status } \\
\hline None $(\%)$ & 6.7 & 1.4 & 53.2 & 16.5 & 18.2 \\
\hline Primary (\%) & 7.5 & 31.5 & 19.0 & 21.2 & 18.2 \\
\hline Junior secondary (\%) & 31.7 & 12.3 & 8.9 & 1.2 & 15.4 \\
\hline Senior Secondary (\%) & 15.0 & 28.8 & 13.9 & 18.8 & 18.5 \\
\hline Tertiary (\%) & 39.2 & 26.0 & 5.1 & 42.4 & 29.7 \\
\hline \multicolumn{6}{|l|}{ Occupation $^{\mathrm{a}}$} \\
\hline Petty trader (\%) & 24.6 & 12.3 & 45.5 & 13.1 & 23.9 \\
\hline JCS (\%) & 23.7 & 19.2 & 6.5 & 3.6 & 14.2 \\
\hline SCS (\%) & 11.0 & 15.1 & 2.6 & 14.3 & 10.8 \\
\hline Businessman (\%) & 11.0 & 20.5 & 22.1 & 17.9 & 17.0 \\
\hline Unemployed (\%) & 4.2 & 5.5 & 15.6 & 1.2 & 6.3 \\
\hline Housewife (\%) & 0.8 & 9.6 & 0 & 1.2 & 2.6 \\
\hline Others (\%) & 1.7 & 9.6 & 2.6 & 0 & 3.1 \\
\hline Artisans (\%) & 5.9 & 0.0 & 1.3 & 7.1 & 4.0 \\
\hline Retired (\%) & 16.9 & 8.2 & 3.9 & 41.7 & 18.2 \\
\hline Use of herbal preparation $(\%)^{\mathrm{a}}$ & 15.8 & 35.6 & 11.7 & 9.4 & 17.5 \\
\hline
\end{tabular}

${ }^{\mathrm{a}} P<0.005$.

BMQ: beliefs about medication questionnaire; IQR: interquartile range; HTN: hypertension; JCS: junior civil servant; P. Harcourt: Port Harcourt; SCS: senior civil servant.

warranted while measures to improve medication adherence are required.

We report a high prevalence of MNA in our study population $(66.7 \%)$, but our finding is within the range of MNA that has been documented in previous studies from sub-Saharan Africa. Most studies in Nigeria and Ghana have documented MNA ranging from 32.7 to $49.3 \%[16,17,20,23]$. There was however a study from Ghana that documented MNA of $93 \%$ before the availability of National Health Insurance [19]. Elsewhere in sub-Saharan Africa, MNA ranged from 10.3 to $87.5 \%$ [32-38]. Variation may be partly due to the different definitions or methods used to assess MNA. Most of the studies though based on self-report, utilized different definitions for MNA. An advantage of our study was that the same tool was used to assess MNA in the various sites. Furthermore we also observed significant association between MNA and BP control in our study which will further validate the tool that was adopted to assess MNA in our study.

Our study found significant correlation between beliefs about medication and adherence to antihypertensives. Patients who had concerns about antihypertensive medications were less likely to be adherent to their medications. These findings are consistent with previous studies on the relationship between medication adherence and beliefs about 
TABLE 2: Correlation of medication adherence (Morisky score) with depression, beliefs about medication, knowledge of hypertension, or income by site and in the pooled data.

\begin{tabular}{lcccccccccc}
\hline \multirow{2}{*}{ Parameter } & \multicolumn{2}{c}{ Korle $\mathrm{Bu}$} & \multicolumn{2}{c}{ Port Harcourt } & \multicolumn{2}{c}{ Lagos } & \multicolumn{2}{c}{ Ibadan } & \multicolumn{2}{c}{ Pooled } \\
& $r$ & $P$ & $r$ & $P$ & $r$ & $P$ & $r$ & $P$ & \multicolumn{1}{c}{$r$} \\
\hline Depression & -0.230 & 0.012 & 0.051 & 0.667 & -0.043 & 0.705 & -0.035 & 0.749 & -0.203 & 0.000 \\
(PHQ-9) & & & & & & & & & \\
BMQ-necessity & 0.142 & 0.124 & -0.079 & 0.507 & 0.091 & 0.433 & 0.002 & 0.984 & -0.087 & 0.103 \\
BMQ-concern & -0.148 & 0.111 & -0.286 & 0.014 & -0.014 & 0.905 & -0.336 & 0.002 & -0.355 & 0.000 \\
BMQ-necessity-concern & 0.215 & 0.020 & 0.174 & 0.141 & 0.079 & 0.505 & 0.210 & 0.060 & 0.337 & 0.000 \\
Hypertension knowledge scale & 0.024 & 0.797 & 0.212 & 0.071 & 0.019 & 0.865 & -0.030 & 0.783 & 0.144 & 0.006 \\
Income & 0.139 & 0.143 & -0.057 & 0.634 & -0.200 & 0.081 & 0.099 & 0.419 & -0.067 & 0.226 \\
\hline
\end{tabular}

BMQ-concern: beliefs about medication concern score; BMQ-necessity: beliefs about medication necessity score; BMQ-necessity-concern: beliefs about medication necessity-concern score; $P=P$ value; $r=$ Pearson's correlation coefficient.

medications [39]. Specifically, patients in our study had concerns about potential adverse effects of long term use of antihypertensives or felt that daily use of antihypertensive medications was a significant disruption of their lifestyles. Patients were also concerned that they may be becoming too dependent on their medications. Other studies have identified side-effects of antihypertensive medications as a reason for MNA in patients $[19,23,35,38]$. Studies have also documented that forgetting to take medications is an important barrier to adherence [17, 23, 38]. In addition, adherence is usually better with medications that require less frequent administration [16, 36-38]. Health education specifically about antihypertensive medications including potential side-effects, and inquiry about side-effects the patient may be experiencing and use of medications that are associated with fewer side effects may lead to improvement in adherence. In addition, use of medications that do not cause more disruption of the patients' daily activities than necessary may also improve adherence.

Our study also found that depression was significantly associated with MNA, particularly among patients at Korle $\mathrm{Bu}$, but also in the pooled data. To the best of our knowledge this study is the first to assess depression and MNA among hypertensives in sub-Saharan Africa. Depression has been associated with poor BP control and medication nonadherence in studies outside sub-Saharan Africa [40, 41]. In a study from the USA, antidepressants were associated with significantly longer initial persistence of antihypertensive therapy among patients with depression who developed hypertension [42]. Evaluation for depression and appropriate management of the depression may improve medication adherence among hypertensives in sub-Saharan Africa.

Patients who were not adherent were significantly younger than patients who were adherent. This pattern has been observed in adherence studies carried outside subSaharan Africa and has also been documented in SubSaharan Africa [26, 43, 44]. The reason for worse medication non adherence in younger patients is not clear. A potential reason may be that younger patients are not as concerned about their health compared with the older patients. Health education directed towards younger age group may improve outcomes among the younger patients.

Our study found positive and significant correlation between knowledge of hypertension and adherence to medications. Previous studies from Africa have associated lack of knowledge regarding the curability of hypertension and lifelong need for antihypertensives with MNA [18, 20, 23, 38]. Our data thus support the need for adequate health education on hypertension to improve MNA in the subregion. Furthermore, patients who reported using herbal preparations for the treatment of hypertension were more likely to show MNA. This is consistent with most studies on hypertension from sub-Saharan Africa that have associated the use of alternative medical therapy with MNA $[17,18,20,36,38]$.

Studies from Nigeria have noted increasing adherence with increasing educational status but these associations were not significant $[16,23]$. Literacy has also been associated with a higher BP control in Nigeria but this was also not significant [45]. Our study, however, found that patients who had received any form of formal education were more likely to show MNA than those who did not. It may be that educated participants in our study were more skeptical towards the use of antihypertensives. A similar observation was noted in a Ghanaian study which also found significant negative association between educational status and medication adherence [17]. Another study from the United States found higher adherence among men with lower levels of education than among more highly educated men [46]. Our data is consistent with a previous suggestion that health education on hypertension and its treatment should be provided for all hypertensive patients in our subregion irrespective of the patients' educational status [47].

In most studies from sub-Saharan Africa participants have identified inability to afford the cost of medications as an important barrier to medication adherence [17-19, 33, 36, 38]. A positive relationship between cost and MNA has also been found in studies outside of Africa [39] Studies have however not consistently demonstrated association of medication adherence with level of income. Our study did not find an association between MNA and income [23, 39, 43]. 
TABLE 3: Distribution of study population characteristics by adherence $(N=357)$.

\begin{tabular}{|c|c|c|c|}
\hline Population Characteristics & $\begin{array}{c}\text { Nonadherent } \\
N(\%)\end{array}$ & $\begin{array}{c}\text { Adherent } \\
N(\%)\end{array}$ & $P$ value \\
\hline Gender & & & 0.623 \\
\hline Male & $104(68.4)$ & $48(31.6)$ & \\
\hline Female & $134(65.4)$ & $71(34.6)$ & \\
\hline Study Site & & & $<0.001$ \\
\hline Korle-Bu & $87(72.5)$ & $33(27.5)$ & \\
\hline Port Harcourt & $70(95.9)$ & $3(4.1)$ & \\
\hline Lagos & $42(53.2)$ & $37(46.8)$ & \\
\hline Ibadan & $39(45.9)$ & $46(54.1)$ & \\
\hline Age & $\begin{array}{c}54.4 \pm 13.2 \\
\text { years }\end{array}$ & $\begin{array}{c}60.9 \pm 12.1 \\
\text { years }\end{array}$ & 0.000 \\
\hline Educational status & & & 0.006 \\
\hline None & $31(47.7)$ & $34(52.3)$ & \\
\hline Primary & $48(73.8)$ & $17(26.2)$ & \\
\hline Junior & $42(76.4)$ & $13(23.6)$ & \\
\hline Secondary & $47(71.2)$ & $19(28.8)$ & \\
\hline Tertiary & $70(60.6)$ & $36(34.0)$ & \\
\hline Formal education & & & 0.001 \\
\hline Yes & $207(70.9)$ & $85(29.1)$ & \\
\hline No & $31(47.7)$ & $34(52.3)$ & \\
\hline Marital status & & & 0.967 \\
\hline Married & $172(66.9)$ & $85(33.1)$ & \\
\hline Not married & $66(66)$ & $34(34)$ & \\
\hline Children & & & 0.04 \\
\hline Yes & $217(65.2)$ & $116(34.8)$ & \\
\hline No & $21(87.5)$ & $3(12.5)$ & \\
\hline Occupation & & & 0.317 \\
\hline Employed & $176(68.5)$ & $81(38.5)$ & \\
\hline Unemployed & $59(62.1)$ & $36(37.9)$ & \\
\hline Cost of drug $(\$)$ & & & 0.1 \\
\hline$<10$ & $58(73.4)$ & $21(26.6)$ & \\
\hline $11-30$ & $87(65.9)$ & $45(34.1)$ & \\
\hline $31-50$ & $36(67.9)$ & $17(32.1)$ & \\
\hline $51-100$ & $27(69.2)$ & $12(30.8)$ & \\
\hline$>100$ & $14(45.2)$ & $17(50.8)$ & \\
\hline Insurance & & & 0.032 \\
\hline Yes & $114(73.1)$ & $42(26.9)$ & \\
\hline No & $124(61.7)$ & $77(38.3)$ & \\
\hline Use of herbal medication & & & 0.014 \\
\hline Herbal med. & $50(80.6)$ & $12(19.4)$ & \\
\hline No herbal med. & $186(63.5)$ & $107(36.5)$ & \\
\hline Hypertension & & & 0.006 \\
\hline Control & $72(56.7)$ & $55(43.3)$ & \\
\hline Uncontrolled & $163(71.8)$ & $64(21.2)$ & \\
\hline Comorbidities & & & 0.874 \\
\hline No comorbidity & $132(69.8)$ & $57(30.2)$ & \\
\hline DM or renal comorbidities & $62(62.6)$ & $37(37.4)$ & \\
\hline Other comorbidities & $38(69.8)$ & $23(30.2)$ & \\
\hline
\end{tabular}

DM: diabetes mellitus.

One explanation may be that in many African studies, as in ours, only a negligible number belong to the high socioeconomic class, making it difficult to access MNA in the high socioeconomic class $[23,45]$. On the other hand the lack of association between income and MNA may reflect an interplay of other factors that contribute to medication adherence. A study carried out in Nigeria in a setting in which antihypertensives were offered for free, noted that BP control was low though higher than in settings where medications were not given for free suggesting that MNA may not be entirely due to cost [48].

We found that access to National Health Insurance was significantly associated with MNA. In addition, BP tended to be higher in patients with access to health insurance than those who did not. Most of the patients who had access to National health Insurance were from Ghana. The Ghanaian patients also had the highest proportion of patients with depression and the depression may have contributed to the high proportion of patients with MNA in the Ghanaian patients in spite of the access to health Insurance. In addition, the way the health insurance system operates may also be contributory. In Ghana for instance, a three-month prescription from clinicians is usually dispensed in three tranches where patients are given a one month supply and asked to come back for refill of their medications for the other two months. Some patients do not return for refill of their medication because of lack of travel funds or lack of a system to remind them to go for the refill. This argues in favour of a critical review of the current national health insurance system in Ghana. Esunge and colleagues in Cameroon had suggested that factors that improved MNA among hypertensives in low resource settings were free medications, free hospital visits, free transportation, open discussion with medical staff, use of common dialects, and politeness of medical staff [49].

Another method that may contribute to improvement in medication adherence in low resource settings is the use of phone technology. Use of text messages has been associated with improvement in adherence to medications [50]. Use of phone technology reminders even in low resource settings may be useful in improving medication adherence in a large number of patients.

Strengths of our study include the multicenter nature of the study, and the use of uniform tools to assess medication non-adherence, depression, knowledge of hypertension, and beliefs about medication across the sites. Limitations of our study are that the study is hospital-based and most patients were from tertiary health centers. As such, the patients in our study are likely to have more comorbidities and to be more highly motivated than patients in the community. Therefore, proportion of patients who are non-adherent in the community may be higher than the observation in our study. Furthermore, MNA was assessed by verbal report and this may also potentially underestimate adherence because of recall bias or social desirability bias, and this may also apply to the other self-report measures. A more objective measure such as urine antihypertensive drug assay, may have demonstrated a higher degree of MNA. However, the study provides valuable information on the burden of MNA among hypertensives and the potential influence of factors such as beliefs about medications, depression, and health insurance on MNA. 


\section{Conclusion}

We studied MNA among hypertensives in hospitals in Ghana and Nigeria. MNA was found in $66.7 \%$ of participants. MNA occurred in younger patients and in patients who had varying degrees of depression or were concerned about their medications. Knowledge of hypertension was positively and significantly correlated with adherence. MNA was associated with use of herbal preparations, and formal education. Expectedly adherence was significantly associated with BP control. We also found that MNA was associated with health insurance, and this may be related to either underlying depression or the mode of operation of the health insurance. The finding of significant association between health insurance and MNA underscores the need for studies to identify the underlying causes of this association. Treatment of depression in patients with hypertension and depression may improve outcomes. Patients irrespective of educational status need education concerning treatment of hypertension and sideeffects of medication in addition to education on hypertension to improve MNA. Other methods to improve medication adherence such as use of phone technology should also be studied.

\section{Conflict of Interests}

The authors declare that there is no conflict of interests regarding the publication of this paper.

\section{Acknowledgments}

Vincent Boima, Adebowale Ademola, Francis Agyekum, Aina Olufemi Odusola, and Eze Nwafor are grateful to the Cardiovascular Research Training Institute (Fogarty International Center Grant no. 5D43TW009140) for training and funding support. The authors are also grateful to Professor Daniel T Lackland, Profssors Kennedy Cruickshank, Dr. Berthelsen Nathan, and Dr Pauline E. Osamor, for support in conceptualizing the study, and to Prof W. Chaplin for assistance with the statistics. The authors appreciate Miss Polina Krass for her assistance with data management.

\section{References}

[1] World Health Organization, Global Health Risks: Mortality and Burden of Disease Attributable to Selected Major Risks, 2009.

[2] G. Danaei, M. M. Finucane, J. K. Lin et al., "National, regional, and global trends in systolic blood pressure since 1980: systematic analysis of health examination surveys and epidemiological studies with 786 country-years and 5.4 million participants," The Lancet, vol. 377, no. 9765, pp. 568-577, 2011.

[3] W. K. Bosu, "Epidemic of hypertension in Ghana: a systematic review," BMC Public Health, vol. 10, article 418, 2010.

[4] C. Agyemang, M. A. Bruijnzeels, and E. Owusu-Dabo, "Factors associated with hypertension awareness, treatment, and control in Ghana, West Africa," Journal of Human Hypertension, vol. 20, no. 1, pp. 67-71, 2006.

[5] A. G. B. Amoah, "Hypertension in Ghana: a cross-sectional community prevalence study in Greater Accra," Ethnicity \& Disease, vol. 13, no. 3, pp. 310-315, 2003.
[6] I. I. Ulasi, C. K. Ijoma, and O. D. Onodugo, "A communitybased study of hypertension and cardio-metabolic syndrome in semi-urban and rural communities in Nigeria," BMC Health Services Research, vol. 10, article 71, 2010.

[7] M. E. Hendriks, F. W. N. M. Wit, M. T. L. Roos et al., "Hypertension in Sub-Saharan Africa: cross-sectional surveys in four rural and urban communities," PLoS ONE, vol. 7, no. 3, Article ID e32638, 2012.

[8] R. A. Adedoyin, C. E. Mbada, M. O. Balogun et al., "Prevalence and pattern of hypertension in a semiurban community in Nigeria," European Journal of Cardiovascular Prevention and Rehabilitation, vol. 15, no. 6, pp. 683-687, 2008.

[9] J. O. Awobusuyi, O. O. Kukoyi, M. A. Ibrahim, and M. Atiba, "Indices of kidney damage and cardiovascular disease risk factors in a semiurban community of Iloye, South-west Nigeria," International Journal of Nephrology, vol. 2011, Article ID 564050, 6 pages, 2011.

[10] E. K. Oghagbon, A. B. Okesina, and S. A. Biliaminu, "Prevalence of hypertension and associated variables in paid workers in Ilorin, Nigeria," Nigerian Journal of Clinical Practice, vol. 11, no. 4, pp. 342-346, 2008.

[11] I. I. Ulasi, C. K. Ijoma, B. J. C. Onwubere, E. Arodiwe, O. Onodugo, and C. Okafor, "High prevalence and low awareness of hypertension in a market population in Enugu, Nigeria," International Journal of Hypertension, vol. 2011, Article ID 869675, 5 pages, 2011.

[12] I. J. Brown, I. Tzoulaki, V. Candeias, and P. Elliott, "Salt intakes around the world: implications for public health," International Journal of Epidemiology, vol. 38, no. 3, pp. 791-813, 2009.

[13] World Health Organization, Global Status Report on Noncommunicable Diseases 2010: Description of the Global Burden of NCDs, Their Risk Factors and Determinants, 2011.

[14] J. Spencer, E. Phillips, and G. Ogedegbe, "Knowledge attitudes, beliefs, and blood pressure control in a community-based sample in Ghana," Ethnicity \& Disease, vol. 15, no. 4, pp. 748$752,2005$.

[15] L. M. Lewis, A. M. Schoenthaler, and G. Ogedegbe, "Patient factors, but not provider and health care system factors, predict medication adherence in hypertensive black men," The Journal of Clinical Hypertension, vol. 14, no. 4, pp. 250-255, 2012.

[16] M. R. Akpa, D. I. Agomuoh, and O. J. Odia, "Drug compliance among hypertensive patients in Port Harcourt, Nigeria," Nigerian Journal of Medicine, vol. 14, no. 1, pp. 55-57, 2005.

[17] T. H. Harries, V. Twumasi-Abosi, J. Plange-Rhule, and F. P. Cappuccio, "Hypertension management in Kumasi: barriers and prejudice?" Journal of Human Hypertension, vol. 19, no. 12, pp. 975-977, 2005.

[18] M. Kabir, Z. Iliyasu, L. Abubakar, and M. Jibril, "Compliance to medication among hypertensive patients in Murtala Mohammed Specialist Hospital, Kano, Nigeria," Journal of Community Medicine and Primary Health Care, vol. 16, no. 1, 2005.

[19] K. O. Buabeng, L. Matowe, and J. Plange-Rhule, "Unaffordable drug prices: the major cause of non-compliance with hypertension medication in Ghana," Journal of Pharmacy and Pharmaceutical Sciences, vol. 7, no. 3, pp. 350-352, 2004.

[20] P. E. Osamor and B. E. Owumi, "Factors associated with treatment compliance in hypertension in southwest Nigeria," Journal of Health, Population and Nutrition, vol. 29, no. 6, pp. 619-628, 2011.

[21] K. B. Yusuff and O. Balogun, "Physicians' prescribing of anti-hypertensive combinations in a tertiary care setting in 
southwestern Nigeria," Journal of Pharmacy \& Pharmaceutical Sciences, vol. 8, no. 2, pp. 235-242, 2005.

[22] A. Oyati, A. Orogade, S. Danbauchi, and P. Azuh, "Awareness, Treatment and Control of Hypertension among Hypertensives in Zaria," Journal of Medicine in the Tropics, vol. 13, no. 2, 2011.

[23] C. O. Amira and N. U. Okubadejo, "Factors influencing noncompliance with anti-hypertensive drug therapy in Nigerians," The Nigerian Postgraduate Medical Journal, vol. 14, no. 4, pp. 325-329, 2007.

[24] J. O. B. Olubodun, A. O. Falase, and T. O. Cole, "Drug compliance in hypertensive Nigerians with and without heart failure," International Journal of Cardiology, vol. 27, no. 2, pp. 229-234, 1990.

[25] A. V. Chobanian, G. L. Bakris, H. R. Black et al., "Seventh report of the Joint National Committee on Prevention, Detection, Evaluation, and Treatment of High Blood Pressure," Hypertension, vol. 42, no. 6, pp. 1206-1252, 2003.

[26] D. E. Morisky, L. W. Green, and D. M. Levine, "Concurrent and predictive validity of a self-reported measure of medication adherence," Medical Care, vol. 24, no. 1, pp. 67-74, 1986.

[27] P. A. James, S. Oparil, B. L. Carter et al., "2014 evidence-based guideline for the management of high blood pressure in adults: report from the panel members appointed to the Eighth Joint National Committee (JNC8)," Journal of the American Medical Association, vol. 311, no. 5, pp. 507-520, 2014.

[28] R. Horne, J. Weinman, and M. Hankins, "The beliefs about medicines questionnaire: the development and evaluation of a new method for assessing the cognitive representation of medication," Psychology and Health, vol. 14, no. 1, pp. 1-24, 1999.

[29] S. Sanne, P. Muntner, L. Kawasaki, A. Hyre, and K. B. Desalvo, "Hypertension knowledge among patients from an urban clinic," Ethnicity and Disease, vol. 18, no. 1, pp. 42-47, 2008.

[30] O. C. Alebiosu, O. B. Familoni, O. O. Ogunsemi et al., "Original research: knowledge of diabetes and hypertension care among health care workers in southwest Nigeria," Postgraduate Medicine, vol. 121, no. 1, pp. 173-177, 2009.

[31] National High Blood Pressure Education Program, The Seventh Report of the Joint National Committee on Prevention, Detection, Evaluation, and Treatment of High Blood Pressure, National Heart, Lung, and Blood Institute, Bethesda, Md, USA, 2004, http://www.ncbi.nlm.nih.gov/books/NBK9630/?report=reader.

[32] N. G. Nkosi and S. C. Wright, "Knowledge related to nutrition and hypertension management practices of adults in GaRankuwa day clinics," Curationis, vol. 33, no. 2, pp. 33-40, 2010.

[33] A. G. Elzubier, A. A. Husain, I. A. Suleiman, and Z. A. Hamid, "Drug compliance among hypertensive patients in Kassala, Eastern Sudan," Eastern Mediterranean Health Journal, vol. 6, no. 1, pp. 100-105, 2000.

[34] D. W. R. Lunt, P. R. Edwards, K. Steyn, C. J. Lombard, and G. S. Fehrsen, "Hypertension care at a Cape Town community health centre," South African Medical Journal, vol. 88, no. 5, pp. 544548, 1998.

[35] M. D. Mweene, J. Banda, B. Andrews, M. M. Mweene, and S. Lakhi, "Factors associated with poor medication adherence in hypertensive patients in Lusaka, Zambia," Medical Journal of Zambia, vol. 37, no. 3, pp. 252-261, 2010.

[36] C. Konin, M. Adoh, I. Coulibaly et al., "Black Africans' compliance to antihypertensive treatment," Archives des Maladies du Coeur et des Vaisseaux, vol. 100, no. 8, pp. 630-634, 2007.

[37] E. E. Maro and J. Lwakatare, "Medication compliance among Tanzanian hypertensives," East African Medical Journal, vol. 74, no. 9, pp. 539-542, 1997.
[38] K. Peltzer, "Health beliefs and prescription medication compliance among diagnosed hypertension clinic attenders in a rural South African Hospital," Curationis, vol. 27, no. 3, pp. 15-23, 2004.

[39] T. Mathes, T. Jaschinski, and D. Pieper, "Adherence influencing factors-a systematic review of systematic reviews," Archives of Public Health, vol. 72, no. 1, p. 37, 2014.

[40] M. Lemstra and M. W. Alsabbagh, "Proportion and risk indicators of nonadherence to antihypertensive therapy: a metaanalysis," Patient Preference and Adherence, vol. 8, pp. 211-218, 2014.

[41] A. F. Rubio-Guerra, L. Rodriguez-Lopez, G. Vargas-Ayala, S. Huerta-Ramirez, D. C. Serna, and J. J. Lozano-Nuevo, "Depression increases the risk for uncontrolled hypertension," Experimental and Clinical Cardiology, vol. 18, no. 1, pp. 10-12, 2013.

[42] J. A. Schmittdiel, W. Dyer, C. Uratsu et al., "Initial persistence with antihypertensive therapies is associated with depression treatment persistence, but not depression," Journal of Clinical Hypertension, vol. 16, no. 6, pp. 412-417, 2014.

[43] P. Bovet, J.-P. Gervasoni, M. Mkamba, M. Balampama, C. Lengeler, and F. Paccaud, "Low utilization of health care services following screening for hypertension in Dar es Salaam (Tanzania): a prospective population-based study," BMC Public Health, vol. 8, article 407, 2008.

[44] M. A. Marentette, W. C. Gerth, D. K. Billings, and K. B. Zarnke, "Antihypertensive persistence and drug class," The Canadian Journal of Cardiology, vol. 18, no. 6, pp. 649-656, 2002.

[45] S. A. Isezuo, A. A. Sabir, A. E. Ohwovorilole, and O. A. Fasanmade, "Prevalence, associated factors and relationship between prehypertension and hypertension: a study of two ethnic African populations in Northern Nigeria," Journal of Human Hypertension, vol. 25, no. 4, pp. 224-230, 2011.

[46] J. Braverman and J. Dedier, "Predictors of medication adherence for African American patients diagnosed with hypertension," Ethnicity and Disease, vol. 19, no. 4, pp. 396-400, 2009.

[47] D. A. Oke and E. O. Bandele, "Misconceptions of hypertension," Journal of the National Medical Association, vol. 96, no. 9, pp. 1221-1224, 2004.

[48] B. L. Salako, F. A. Ajose, and E. Lawani, "Blood pressure control in a population where antihypertensives are given free," East African Medical Journal, vol. 80, no. 10, pp. 529-531, 2003.

[49] P. M. Esunge, "Patient compliance and the evaluation of drug trials for hypertension in rural Africa," Ethnicity \& Disease, vol. 1, no. 3, pp. 292-294, 1991.

[50] S. Krishna, S. A. Boren, and E. A. Balas, "Healthcare via cell phones: a systematic review," Telemedicine and e-Health, vol. 15, no. 3, pp. 231-240, 2009. 


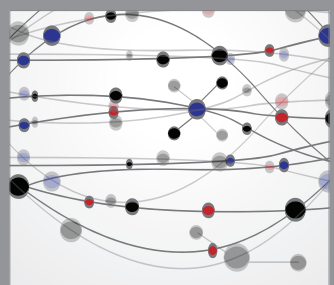

The Scientific World Journal
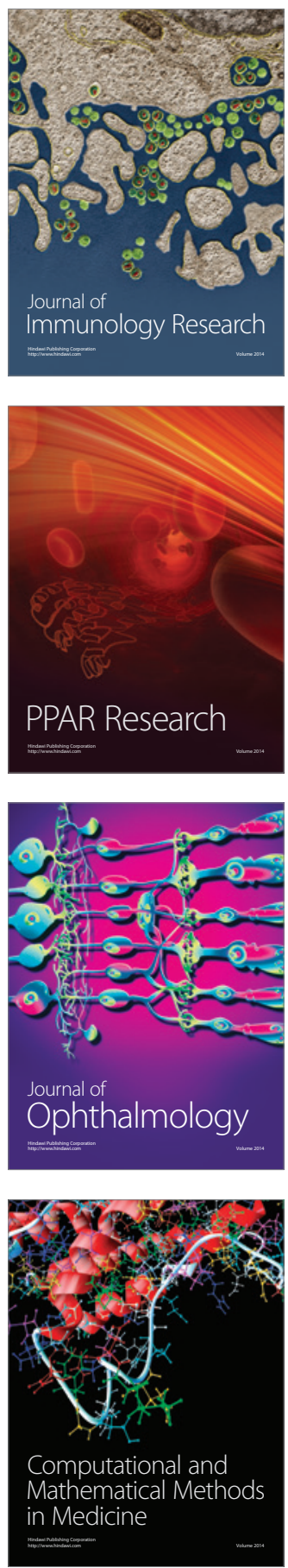

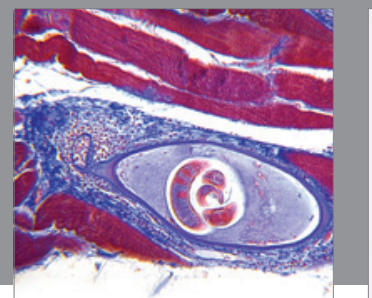

Gastroenterology

Research and Practice
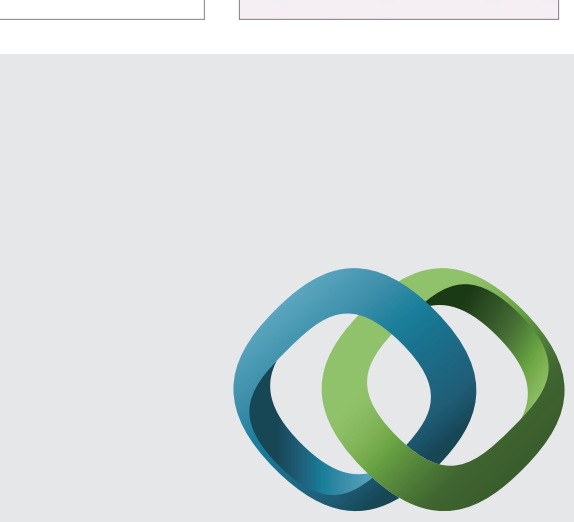

\section{Hindawi}

Submit your manuscripts at

http://www.hindawi.com
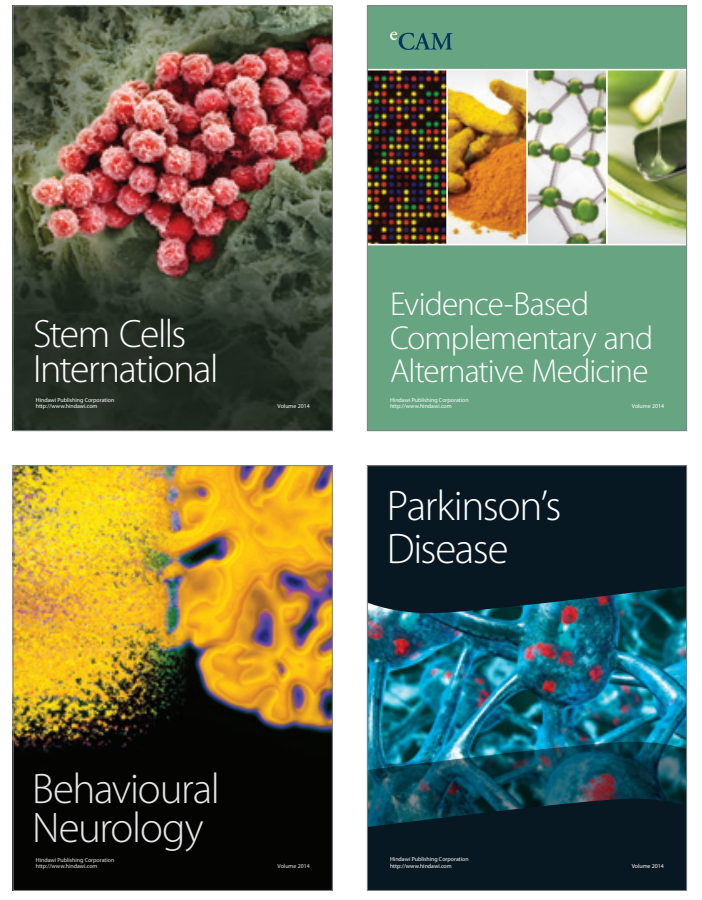
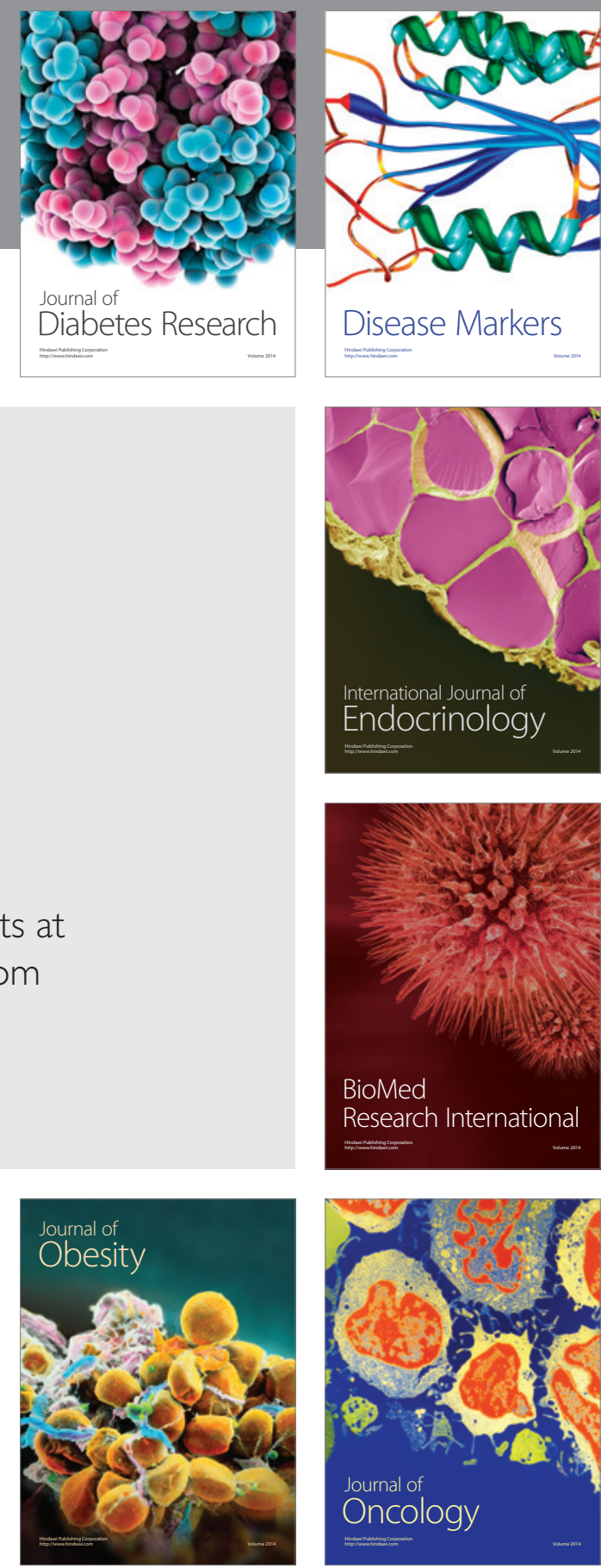

Disease Markers
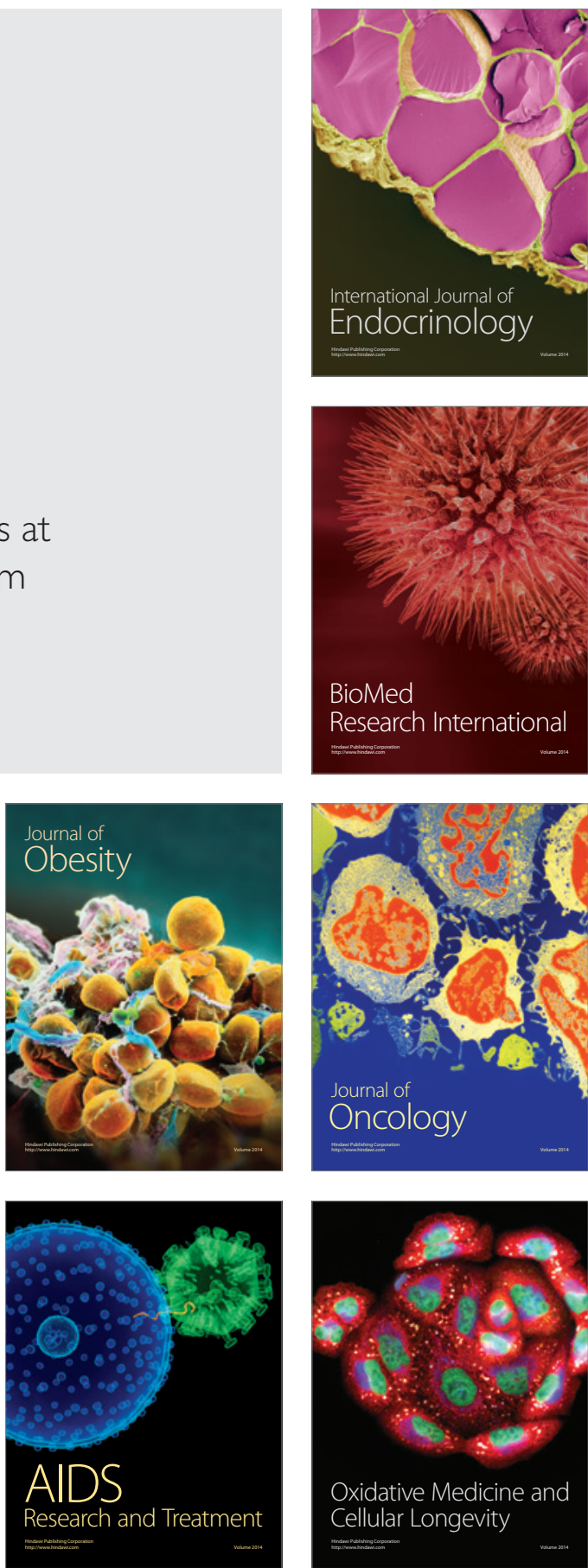